\title{
Eficácia de herbicidas isolados e em associações no controle em pós-emergência de plantas daninhas
}

\author{
Effectiveness of herbicides isolated and in associations in the control at \\ post-emergence of weeds
}

\begin{abstract}
André Felipe Moreira Silva ${ }^{1 *}$, Ana Ligia Giraldeli ${ }^{1}$, Felipe Carrara de Brito ${ }^{1}$, Ana Carolina Viviani Pagenotto ${ }^{1}$, Júlia Pereira de Moraes ${ }^{1}$, Maiara Maria Franzoni ${ }^{1}$, Ricardo Victoria Filho ${ }^{1}$
\end{abstract}

Resumo - É essencial a rotação de mecanismos de ação de herbicidas no manejo de plantas daninhas resistentes ao glyphosate, neste contexto o uso de herbicidas inibidores da enzima ALS em associação pode auxiliar no controle. O objetivo do trabalho foi avaliar a eficácia de sulfometuron, chlorimuron e outras sulfonilureias, isolados ou em associação com glyphosate, no controle de amendoim-bravo, corda-de-viola e picão-preto. Foram realizados três experimentos em casa-de-vegetação, em delineamento inteiramente casualizado, com quatro repetições. Os tratamentos corresponderam aos herbicidas aplicados em pós-emergência das plantas daninhas mais a testemunha (sem aplicação), quando estas estavam com duas folhas completamente desenvolvidas. Os resultados evidenciaram que sulfometuron ( $15 \mathrm{~g} \mathrm{ha}^{-1}$ i.a. $)+$ chlorimuron $\left(20 \mathrm{~g} \mathrm{ha}^{-1}\right.$ i.a. $)+$ glyphosate $\left(960 \mathrm{~g} \mathrm{ha}^{-1}\right.$ e.a. $)$, foi eficaz no controle de amendoim-bravo, e o tratamento halosulfuron $\left(80 \mathrm{~g} \mathrm{ha}^{-1}\right.$ i.a. $)+$ glyphosate ( $960 \mathrm{~g} \mathrm{ha}^{-1}$ e.a.) propiciou controle de $76,25 \%$. A aplicação de sulfometuron $\left(15 \mathrm{~g} \mathrm{ha}^{-1}\right.$ i.a. $)+$ chlorimuron $\left(20 \mathrm{~g} \mathrm{ha}^{-1}\right.$ i.a. $)+$ glyphosate $\left(960 \mathrm{~g} \mathrm{ha}^{-1}\right.$ e.a. $)$, foi eficaz no controle de corda-de-viola. Todos os tratamentos herbicidas foram eficazes no controle em pós-emergência de picão-preto.

Palavras-chave: sulfonilureias, manejo químico, associação de herbicidas, Euphorbia heterophylla, Ipomoea purpurea, Bidens subalternans

Abstract - It is essential the rotation of mechanisms of action in the management of glyphosate resistant weeds, in this context the use of ALS inhibitor herbicides in association can aid in the control. The aim of this work was to evaluate the efficacy of sulfometuron, chlorimuron and other sulfonylureas, isolated and in associations in the control of Wild Poinsettia, Morning Glory and Greater Beggarticks. Three greenhouse experiments were carried out in a completely randomized design with four replications. The treatments corresponded to the herbicides applied in post-emergence of the weed plus the control (without application), when these were with two completely developed leaves. The results showed that sulfometuron $\left(15 \mathrm{~g} \mathrm{ha}^{-1}\right.$ a.i. $)+$ chlorimuron $\left(20 \mathrm{~g} \mathrm{ha}^{-1} \mathrm{a}\right.$ a.i. $)+$ glyphosate ( $960 \mathrm{~g} \mathrm{ha}^{-1}$ a.e.) was effective in the control of Wild Poinsettia, and halosulfuron treatment ( $80 \mathrm{~g} \mathrm{ha}^{-1}$ a.i.) + glyphosate $\left(960 \mathrm{~g} \mathrm{ha}^{-1}\right.$ a.e.) provided a control of $76.25 \%$. The application of sulfometuron $\left(15 \mathrm{~g} \mathrm{ha}^{-1}\right.$ a.i. $)+$ chlorimuron $\left(20 \mathrm{~g} \mathrm{ha}^{-1}\right.$ a.i. $)+$ glyphosate $\left(960 \mathrm{~g} \mathrm{ha}^{-1}\right.$ a.e. $)$ was effective in the control of Morning Glory. All herbicides treatments were effective in the post-emergence control of Greater Beggarticks.

Keywords: sulfonylureas, chemical management, association of herbicides, Euphorbia heterophylla, Ipomoea purpurea, Bidens subalternans

Recebido: Setembro 12, 2017. Aceito: Julho 16, 2018.

${ }^{1}$ Escola Superior de Agricultura "Luiz de Queiroz" - ESALQ, Universidade de São Paulo - USP, Avenida Pádua Dias, 11, CEP: 13418-900, Piracicaba, SP, Brasil. E-mail: afmoreirasilva@hotmail.com, analigia_giraldeli@hotmail.com, britofelipe@hotmail.com, ana.pagenotto@gmail.com, juliapmoraes@usp.br, maiarafranzoni@gmail.com, rvictori@usp.br 


\section{Introdução}

O controle químico constitui-se de importante ferramenta no manejo de plantas daninhas. Assim, os herbicidas podem ser aplicados antes do plantio, em pré-plantio incorporado (PPI), pré-emergência (PRÉ) e em pós-emergência (PÓS) da cultura e das plantas daninhas (Constantin, 2011).

Com o desenvolvimento dos materiais tolerantes a glyphosate (Roundup Ready ${ }^{\circledR} \mathrm{RR}^{\circledR}$ ), esse herbicida passou a ser o mais utilizado em cultivos de grãos, contudo outros herbicidas, como as sulfonilureias, tem sua importância e devem ser considerados visando ao manejo de plantas daninhas e a prevenção da seleção de biótipos resistentes ao glyphosate. Shaner (2000) já alertava que mesmo o glyphosate sendo importante no manejo de plantas daninhas, o uso intensivo deste herbicida nas culturas $\mathrm{RR}^{\circledR}$ pode acarretar problemas, principalmente na seleção de biótipos resistentes.

Muitos são os casos de biótipos resistentes ao glyphosate pelo mundo, especificamente no Brasil são relatados casos de resistência para as plantas daninhas buva (Conyza sp.), capim-amargoso (Digitaria insularis), azevém (Lolium multiflorum), capim-branco (Chloris elata), capim-pé-de-galinha (Eleusine indica) e caruru (Amaranthus palmeri) (Heap, 2018).

Assim a associação e rotação de herbicidas, de diferentes mecanismos de ação, devem ser consideradas no manejo de plantas daninhas de difícil controle (Riar et al., 2013; Gazziero, 2015). Nesse contexto a soja tolerante a sulfonilureias $\left(\mathrm{STS}^{\circledR}\right)$ merece destaque pela possibilidade de uso de herbicidas do grupo das sulfonilureias em doses acima das recomendadas, ou até mesmo de herbicidas que não eram seletivos para cultivares não-STS (Green, 2007; Roso e Vidal, 2011; Silva et al., 2016; Albrecht et al., 2017). Estes herbicidas inibem a enzima ALS e controlam principalmente plantas daninhas eudicotiledôneas e são utilizados nas culturas do trigo, arroz, soja, cevada, algodão, batata e milho (Zhou et al., 2007).
As plantas daninhas eudicotiledôneas amendoim-bravo (Euphorbia heterophylla), corda-de-viola (Ipomoea purpurea) e picão-preto (Bidens subalternans) estão presentes em diversos cultivos no Brasil, com importância nos cultivos de grãos e cana-de-açúcar (Kissmann e Groth, 2007; Moreira e Bragança, 2011).

Pagnoncelli et al. (2017) observaram reduções de cerca de $26 \%$ na produtividade de soja, em competição com uma planta de Ipomoea sp. $\mathrm{m}^{-2}$. Por sua vez Ferreira et al. (2015) obervaram reduções em massa seca da parte áerea e parâmetros fotossintéticos de soja em competição com picão-preto (Bidens pilosa). Forte et al. (2017) verificaram reduções na área foliar e massa seca de parte de soja em competição com amendoim-bravo. O herbicida sulfometuron apresenta registro no Brasil como maturador para uso na cultura da cana-de-açúcar. Também apresenta registro, em misturas comerciais com outros herbicidas, para o controle de plantas daninhas na cultura da cana-de-açúcar (Brasil, 2017). Na argentina é utilizado em mistura pronta de fábrica com chlorimuron para controle de plantas daninhas em soja STS ${ }^{\circledR}$ (Olea, 2013). No Brasil a mistura comercial de sulfometuron e chlorimuron encontra-se em fase registro. Assim acredita-se que sulfometuron, chlorimuron e outras sulfonilureias, isoladas ou em associação com glyphosate, podem ser eficazes no controle de plantas daninhas eudicotiledôneas.

Portanto, objetivou-se com o presente trabalho, avaliar a eficácia de sulfometuron, chlorimuron e outras sulfonilureias, isolados ou em associação com glyphosate, no controle de amendoim-bravo, corda-de-viola e picão-preto.

\section{Material e métodos}

Foram conduzidos três experimentos em casa de vegetação, nos meses de junho e julho de 2017. Foram utilizadas as plantas daninhas amendoim-bravo, corda-de-viola e picão-preto, experimentos I, II e III respectivamente. 
Os tratamentos foram compostos da aplicação, em pós-emergência das plantas daninhas dos herbicidas sulfometuron (Curavial ${ }^{\circledR}, 750 \mathrm{~kg}^{-1}$ g i.a., WG, Du Pont), chlorimuron (Classic ${ }^{\circledR}, 250 \mathrm{~kg}^{-1} \mathrm{~g}$ i.a., WG, Du Pont), ethoxysulfuron (Gladium ${ }^{\circledR}, 600 \mathrm{~kg}^{-1} \mathrm{~g}$ i.a., WG, Bayer), halosulfuron (Sempra ${ }^{\circledR}, 750 \mathrm{~kg}^{-1} \mathrm{~g}$ i.a., WG, Arysta) e glyphosate (Roundup Ready ${ }^{\circledR}$, $480 \mathrm{~L}^{-1} \mathrm{~g}$ e.a., CS, Monsanto), tanto isoladamente como em associações (Tabela 1). Foi utilizado delineamento inteiramente casualizado, com quatro repetições e as unidades experimentais foram constituídas por vasos de $3 \mathrm{~L}$.

Os vasos foram preenchidos com solo proveniente de área experimental, da camada de 0-20 $\mathrm{cm}$ de profundidade. A análise física e química do solo utilizado apresentou os seguintes resultados: $\mathrm{pH}\left(\mathrm{CaCl}_{2}\right)=5,3 ; \mathrm{Al}=<1,0 \mathrm{mmol}_{\mathrm{c}} \mathrm{dm}^{-3}$, $\mathrm{H}+\mathrm{Al}=25,0 \mathrm{mmol}_{\mathrm{c}} \mathrm{dm}^{-3}, \mathrm{P}($ resina $)=7,0 \mathrm{mg} \mathrm{dm}^{-3}$; $\mathrm{K}=2,6 \mathrm{mmol}_{\mathrm{c}} \mathrm{dm}^{-3} ; \mathrm{Ca}=39,0 \mathrm{mmol}_{\mathrm{c}} \mathrm{dm}^{-3}$; $\mathrm{Mg}=16,0 \mathrm{mmol}_{\mathrm{c}} \mathrm{dm}^{-3} ; \mathrm{SB}=47,6 \mathrm{mmol}_{\mathrm{c}} \mathrm{dm}^{-3}$, $\mathrm{V}(\%)=70$. Com teores de argila, areia e silte total de: $40 ; 54$ e $6 \%$, respectivamente. Os vasos de cada experimento foram semeados com as respectivas plantas daninhas, após a emergência das plantas realizou-se desbaste, deixando-se uma planta por vaso.

Tabela 1. Tratamentos compostos pela aplicação de sulfometuron, chlorimuron e outros herbicidas, isolados e associados (2017).

\begin{tabular}{lc}
\hline \multicolumn{1}{c}{ Tratamentos } & Doses $^{2}$ \\
\hline 1. testemunha & - \\
2. sulfometuron & 15 \\
3. chlorimuron & 20 \\
4. sulfometuron + chlorimuron & $15+20$ \\
5. ethoxysulfuron & 60 \\
6. halosulfuron & 80 \\
7. sulfometuron + glyphosate & $15+960$ \\
8. chlorimuron + glyphosate & $20+960$ \\
9. sulfometuron + chlorimuron + & $15+20+960$ \\
glyphosate & \\
10. ethoxysulfuron + glyphosate & $60+960$ \\
11. halosulfuron + glyphosate & $80+960$ \\
\hline
\end{tabular}

${ }^{2}$ Gramas por hectare de ingrediente ativo (g ha ${ }^{-1}$ i.a.). Doses por hectare em gramas de equivalente ácido ( $\mathrm{g} \mathrm{ha}^{-1}$ e.a.), para o herbicida glyphosate.
A aplicação dos tratamentos ocorreu quando as plantas atingiram o estádio de 2-3 folhas expandidas. Foi realizada via pulverizador costal pressurizado a $\mathrm{CO}_{2}$, com barra equipada com quatro pontas de pulverização, a uma pressão constante de $200 \mathrm{kPa}$, vazão de $0,65 \mathrm{~L} \mathrm{min.} .^{-1}$, trabalhando a uma altura de $50 \mathrm{~cm}$ do alvo, e a uma velocidade de $1 \mathrm{~m} \mathrm{~s}^{-1}$, atingindo uma faixa aplicada de $50 \mathrm{~cm}$ de largura por ponta de pulverização, e propiciando um volume de calda de $200 \mathrm{~L} \mathrm{ha}^{-1}$.

Foi realizada avaliação de controle aos 7, 14, 21 e 28 dias após aplicação (DAA), considerando $0 \%$ como ausência de controle e $100 \%$ a morte da planta (Velini et al., 1995). Foi também mensurada a massa seca de todo material vegetal remanescente da parte aérea das plantas aos 28 DAA. O material foi coletado e seco em estufa com ventilação forçada por $72 \mathrm{~h}$ a $65^{\circ} \mathrm{C}$ e posteriormente mensurou-se a massa seca em balança analítica, com precisão de duas casas decimais. Ressalta-se que para os dados de controle para os experimentos I e II, e massa seca das plantas daninhas para o experimento III, foi necessário utilizar a opção de transformação $\sqrt{ }(\mathrm{X}+1)$.

Os dados obtidos foram submetidos à análise de variância, as médias dos tratamentos foram comparadas pelo teste de Tukey $(\mathrm{p}<0,05)$, conforme Pimentel-Gomes e Garcia (2002), através do programa estatístico Sisvar (Ferreira, 2014).

\section{Resultados e discussão}

A seguir são apresentados os resultados para o experimento I (Tabela 2). Verifica-se que a aplicação de sulfometuron (15 $\mathrm{g} \mathrm{ha}^{-1}$ i.a.) + chlorimuron (20 g ha ${ }^{-1}$ i.a.) + glyphosate (960 $\mathrm{g} \mathrm{ha}^{-1}$ e.a.) propiciou controle superior a todos os demais tratamentos, aos 21 e 28 DAA, para as plantas de amendoim-bravo. Atingindo 90,00\% de controle aos 28 DAA, ressalta-se ainda a redução na massa seca das plantas, em comparação à testemunha sem aplicação.

Destaca-se também que aplicação de halosulfuron $\left(80 \mathrm{~g} \mathrm{ha}^{-1}\right.$ i.a. $)+$ glyphosate, propiciou controle de $76,25 \%$ aos 28 DAA, inferior apenas ao 
Tabela 2. Controle (\%) aos 7, 14, 21 e 28 DAA e massa seca (mg) de amendoim-bravo (experimento I) (2017).

\begin{tabular}{|c|c|c|c|c|c|}
\hline \multirow{2}{*}{ Tratamentos $^{1}$} & \multicolumn{4}{|c|}{ Controle (DAA) } & \multirow{2}{*}{ Massa seca } \\
\hline & $7^{\mathrm{ns}}$ & $14 *$ & $21 *$ & $28^{*}$ & \\
\hline 1. TEST & 0,00 & $0,00 \mathrm{~b}$ & $0,00 \mathrm{c}$ & $0,00 \mathrm{~d}$ & $150,00 \mathrm{~d}$ \\
\hline 2. SUL & 0,00 & $0,00 \mathrm{~b}$ & $0,00 \mathrm{c}$ & $1,50 \mathrm{~d}$ & $127,50 \mathrm{~cd}$ \\
\hline 3. CHL & 0,00 & $0,00 \mathrm{~b}$ & $0,00 \mathrm{c}$ & $0,00 \mathrm{~d}$ & $147,50 \mathrm{~d}$ \\
\hline 4. SUL + CHL & 0,00 & $0,00 \mathrm{~b}$ & $0,00 \mathrm{c}$ & $5,00 \mathrm{~cd}$ & $105,00 \mathrm{bcd}$ \\
\hline 5. ETH & 0,00 & $0,00 \mathrm{~b}$ & $0,00 \mathrm{c}$ & $0,00 \mathrm{~d}$ & $130,00 \mathrm{~cd}$ \\
\hline 6. HAL & 0,00 & $0,00 \mathrm{~b}$ & $0,00 \mathrm{c}$ & $0,00 \mathrm{~d}$ & $133,00 \mathrm{~cd}$ \\
\hline 7. SUL + GLY & 0,00 & $7,50 \mathrm{ab}$ & $7,50 \mathrm{c}$ & $12,50 \mathrm{c}$ & $87,50 \mathrm{abc}$ \\
\hline 8. CHL + GLY & 0,00 & $0,00 \mathrm{~b}$ & $0,00 \mathrm{c}$ & $0,00 \mathrm{~d}$ & $120,00 \mathrm{~cd}$ \\
\hline 9. SUL + CHL + GLY & 2,50 & $25,00 \mathrm{a}$ & $77,50 \mathrm{a}$ & $90,00 \mathrm{a}$ & $30,00 \mathrm{a}$ \\
\hline 10. ETH + GLY & 0,00 & $3,00 \mathrm{~b}$ & $0,75 \mathrm{c}$ & $0,00 \mathrm{~d}$ & $117,50 \mathrm{~cd}$ \\
\hline 11. HAL + GLY & 2,50 & $9,50 a b$ & $25,00 \mathrm{~b}$ & $76,25 \mathrm{~b}$ & $47,50 \mathrm{ab}$ \\
\hline Média & 0,45 & 4,09 & 10,07 & 16,84 & 108,65 \\
\hline DMS & 4,25 & 18,27 & 12,44 & 7,98 & 55,67 \\
\hline C.V. $(\%)$ & 38,52 & 40,17 & 17,06 & 9,38 & 20,98 \\
\hline
\end{tabular}

${ }^{1}$ TEST $=$ testemunha; $\mathrm{SUL}=$ sulfometuron $\left(15 \mathrm{~g} \mathrm{ha}^{-1}\right.$ i.a. $) ; \mathrm{CHL}=$ chlorimuron $\left(20 \mathrm{~g} \mathrm{ha}^{-1}\right.$ i.a. $)$; ETH $=$ ethoxysulfuron (60 $\mathrm{g} \mathrm{ha}^{-1}$ i.a.); $\mathrm{HAL}=$ halosulfuron $\left(80 \mathrm{~g} \mathrm{ha}^{-1}\right.$ i.a. $) ; \mathrm{GLY}=$ glyphosate $\left(960 \mathrm{~g} \mathrm{ha}^{-1} \mathrm{e} . \mathrm{a}.\right)$; ${ }^{\text {ns }}$ Médias não diferem entre si pelo teste Tukey, ao nível de $5 \%$ de probabilidade; *Médias seguidas pela mesma letra na coluna não diferem entre si pelo teste Tukey, ao nível de 5\% de probabilidade. DAA = dias após a aplicação.

valor verificado para a associação tripla. Demais tratamentos não apresentaram controles satisfatórios de amendoim-bravo, com porcentagens chegando a no máximo 12,5 , aos 28 DAA.

A aplicação de sulfometuron + chlorimuron + glyphosate também apresentou os melhores resultados de controle e redução de matéria seca para corda-de-viola (Tabela 3). Com resultados de controle superiores a todos os demais tratamentos aos 7, 21 e 28 DAA. Demais tratamentos não foram eficazes no controle, aos 28 DAA valores de controle de no máximo $25 \%$.

Enquanto que para o experimento III (Tabela 4) todos os tratamentos herbicidas foram eficazes no controle de picão-preto, bem como reduziram a massa seca em relação à testemunha sem aplicação. Verifica-se controle superior a $93 \%$ aos 21 DAA, para todos os tratamentos herbicidas. Aos 28 DAA o controle atingiu $100 \%$.

A aplicação de halosulfuron + glyphosate apresentou-se como alternativa no manejo de plantas daninhas, por apresentar controle de $76,5 \%$ para amendoim-bravo e 100\% para picão-preto. O herbicida halosulfuron apresenta registro, no Brasil, para controle de Cyperus sp., na dose de 112,5 g ha $^{-1}$ i.a., na cultura da cana-de-açúcar (Rodrigues e Almeida, 2011). A aplicação deste herbicida, isolado ou em associações, também é relatada como eficaz no controle de outras plantas, tais como Erechtites hieraciifolia (Atkinson et al., 2014), Armoracia rusticana (Johanning et al., 2016), Chenopodium album, Amaranthus retroflexus e Ambrosia artemissifolia (Li et al., 2016).

Outro ponto a ser ressaltado é que o herbicida halosulfuron ( $69 \mathrm{~g} \mathrm{ha}^{-1}$ i.a.) apresentou-se tolerante para soja STS ${ }^{\circledR}$ (Nandula et al., 2009). Portanto, devido a sua eficácia no controle de muitas plantas daninhas e sua seletividade para cultivares $\mathrm{STS}^{\circledR}$, pode vir a ser empregado em associação com glyphosate no manejo de plantas daninhas em soja $\mathrm{RR}^{\circledast} / \mathrm{STS}^{\circledR}$.

Destaca-se a eficácia de sulfometuron + chlorimuron + glyphosate no controle das três plantas daninhas estudadas. Nonemacher et al. (2017) 
Tabela 3. Controle (\%) aos 7, 14, 21 e 28 DAA e massa seca (mg) de corda-de-viola (experimento II) (2017).

\begin{tabular}{|c|c|c|c|c|c|}
\hline \multirow{2}{*}{ Tratamentos $^{1}$} & \multicolumn{4}{|c|}{ Controle (DAA) } & \multirow{2}{*}{ Massa seca } \\
\hline & $7 *$ & $14 *$ & $21 *$ & $28 *$ & \\
\hline 1. TEST & $0,00 \mathrm{~b}$ & $0,00 \mathrm{e}$ & $0,00 \mathrm{~d}$ & $0,00 \mathrm{e}$ & $235,00 \mathrm{~b}$ \\
\hline 2. SUL & $3,25 \mathrm{~b}$ & 6,25 cde & $3,75 \mathrm{~cd}$ & $3,25 \mathrm{de}$ & $210,00 \mathrm{ab}$ \\
\hline 3. CHL & $3,00 \mathrm{~b}$ & $23,75 \mathrm{ab}$ & $32,50 \mathrm{~b}$ & $13,75 \mathrm{bcd}$ & $202,50 \mathrm{ab}$ \\
\hline 4. SUL + CHL & $5,00 \mathrm{~b}$ & $17,50 \mathrm{abc}$ & $30,00 \mathrm{~b}$ & $13,75 \mathrm{bcd}$ & $185,00 \mathrm{ab}$ \\
\hline 5. ETH & $6,25 \mathrm{~b}$ & 11,25 cde & $22,50 \mathrm{bc}$ & 10,00 cde & $187,50 \mathrm{ab}$ \\
\hline 6. HAL & $6,25 \mathrm{~b}$ & $15,00 \mathrm{abcd}$ & $18,75 \mathrm{bcd}$ & $5,50 \mathrm{de}$ & $215,00 \mathrm{~b}$ \\
\hline 7. SUL + GLY & $4,50 \mathrm{~b}$ & $13,75 \mathrm{bcd}$ & $22,50 \mathrm{bc}$ & $17,50 \mathrm{bc}$ & $162,50 \mathrm{ab}$ \\
\hline 8. CHL + GLY & $5,00 \mathrm{~b}$ & $15,00 \mathrm{abcd}$ & $26,25 \mathrm{~b}$ & $13,00 \mathrm{~cd}$ & $192,50 \mathrm{ab}$ \\
\hline 9. $\mathrm{SUL}+\mathrm{CHL}+\mathrm{GLY}$ & $17,50 \mathrm{a}$ & $26,25 \mathrm{a}$ & $77,50 \mathrm{a}$ & $83,75 \mathrm{a}$ & $100,00 \mathrm{a}$ \\
\hline 10. ETH + GLY & $6,25 \mathrm{~b}$ & $5,00 \mathrm{de}$ & $4,00 \mathrm{~cd}$ & $4,50 \mathrm{de}$ & $240,00 \mathrm{~b}$ \\
\hline 11. HAL + GLY & $5,75 \mathrm{~b}$ & $13,75 \mathrm{bcd}$ & $30,00 \mathrm{~b}$ & $25,00 \mathrm{~b}$ & $142,50 \mathrm{ab}$ \\
\hline Média & 5,66 & 13,41 & 24,34 & 17,27 & 188,41 \\
\hline DMS & 6,46 & 11,54 & 21,30 & 11,72 & 23,33 \\
\hline C.V. $(\%)$ & 23,06 & 17,52 & 20,86 & 17,67 & 24,77 \\
\hline
\end{tabular}

${ }^{1}$ TEST $=$ testemunha; $\mathrm{SUL}=$ sulfometuron $\left(15 \mathrm{~g} \mathrm{ha}^{-1}\right.$ i.a. $) ; \mathrm{CHL}=$ chlorimuron $\left(20 \mathrm{~g} \mathrm{ha}^{-1}\right.$ i.a. $)$; $\mathrm{ETH}=$ ethoxysulfuron (60 $\mathrm{g} \mathrm{ha}^{-1}$ i.a.); $\mathrm{HAL}=$ halosulfuron ( $80 \mathrm{~g} \mathrm{ha}^{-1}$ i.a.); GLY: glyphosate $\left(960 \mathrm{~g} \mathrm{ha}^{-1}\right.$ e.a.); *Médias seguidas pela mesma letra na coluna não diferem entre si pelo teste Tukey, ao nível de $5 \%$ de probabilidade; DAA= dias após a aplicação; DMS = diferença mínima significativa; C.V. $(\%)=$ coeficiente de variação.

Tabela 4. Controle (\%) aos 7, 14, 21 e 28 DAA e massa seca (mg) de picão-preto (experimento III) (2017).

\begin{tabular}{lccccc}
\hline \multirow{2}{*}{ Tratamentos $^{\mathbf{1}}$} & \multicolumn{4}{c}{ Controle (DAA) } & \multirow{2}{*}{ Massa seca } \\
\cline { 2 - 5 } & $\mathbf{7}^{*}$ & $\mathbf{1 4}$ & $\mathbf{2 1}$ & $\mathbf{2 8}$ & \\
1. TEST & $0,00 \mathrm{~b}$ & $0,00 \mathrm{c}$ & $0,00 \mathrm{c}$ & $0,00 \mathrm{~b}$ & $147,50 \mathrm{~b}$ \\
2. SUL & $30,00 \mathrm{a}$ & $75,00 \mathrm{ab}$ & $98,50 \mathrm{a}$ & $100,00 \mathrm{a}$ & $25,00 \mathrm{a}$ \\
3. CHL & $20,00 \mathrm{ab}$ & $75,00 \mathrm{ab}$ & $98,00 \mathrm{ab}$ & $100,00 \mathrm{a}$ & $40,00 \mathrm{a}$ \\
4. SUL + CHL & $41,25 \mathrm{a}$ & $90,50 \mathrm{a}$ & $98,50 \mathrm{a}$ & $100,00 \mathrm{a}$ & $7,50 \mathrm{a}$ \\
5. ETH & $20,00 \mathrm{ab}$ & $67,50 \mathrm{~b}$ & $93,50 \mathrm{~b}$ & $100,00 \mathrm{a}$ & $42,50 \mathrm{a}$ \\
6. HAL & $20,00 \mathrm{ab}$ & $78,75 \mathrm{ab}$ & $97,50 \mathrm{ab}$ & $100,00 \mathrm{a}$ & $20,00 \mathrm{a}$ \\
7. SUL + GLY & $40,00 \mathrm{a}$ & $82,50 \mathrm{ab}$ & $95,00 \mathrm{ab}$ & $100,00 \mathrm{a}$ & $40,00 \mathrm{a}$ \\
8. CHL + GLY & $25,00 \mathrm{ab}$ & $70,00 \mathrm{ab}$ & $95,00 \mathrm{ab}$ & $100,00 \mathrm{a}$ & $27,50 \mathrm{a}$ \\
9. SUL + CHL + GLY & $40,00 \mathrm{a}$ & $90,00 \mathrm{a}$ & $99,00 \mathrm{a}$ & $100,00 \mathrm{a}$ & $20,00 \mathrm{a}$ \\
10. ETH + GLY & $25,00 \mathrm{ab}$ & $87,50 \mathrm{ab}$ & $98,50 \mathrm{a}$ & $100,00 \mathrm{a}$ & $20,00 \mathrm{a}$ \\
11. HAL + GLY & $32,50 \mathrm{a}$ & $91,50 \mathrm{a}$ & $98,50 \mathrm{a}$ & $100,00 \mathrm{a}$ & $15,00 \mathrm{a}$ \\
Média & 26,70 & 73,48 & 88,36 & 90,91 & 36,82 \\
DMS & 26,53 & 21,59 & 4,77 & 0,00 & 45,59 \\
C.V. (\%) & 40,68 & 12,03 & 2,21 & 0,00 & 0,90 \\
\hline
\end{tabular}

${ }^{1}$ TEST $=$ testemunha; $\mathrm{SUL}=$ sulfometuron $\left(15 \mathrm{~g} \mathrm{ha}^{-1}\right.$ i.a. $) ; \mathrm{CHL}=$ chlorimuron $\left(20 \mathrm{~g} \mathrm{ha}^{-1}\right.$ i.a. $)$; ETH $=$ ethoxysulfuron (60 $\mathrm{g} \mathrm{ha}^{-1}$ i.a.); $\mathrm{HAL}=$ halosulfuron $\left(80 \mathrm{~g} \mathrm{ha}^{-1}\right.$ i.a. $) ; \mathrm{GLY}=$ glyphosate $\left(960 \mathrm{~g} \mathrm{ha}^{-1}\right.$ e.a. $)$; *Médias seguidas pela mesma letra na coluna não diferem entre si pelo teste Tukey, ao nível de $5 \%$ de probabilidade; DAA= dias após a aplicação; DMS = diferença mínima significativa; C.V. $(\%)=$ coeficiente de variação.

verificaram controle de $88 \%$ de Urochloa plantaginea e Digitaria ciliaris, aos 21 DAA, para aplicação de sulfometuron $\left(15 \mathrm{~g} \mathrm{ha}^{-1}\right.$ i.a. $)+$ chlorimuron (20 $\mathrm{g} \mathrm{ha}^{-1}$ i.a.) em pré-emergência, seguida da aplicação em pós- emergência de glyphosate (1080 $\mathrm{g} \mathrm{ha}^{-1}$ e.a.). De modo semelhante Piasecki e Rizzardi (2016) verificaram controle superior a $95 \%$ de milho $\mathrm{RR}^{\circledast}$ voluntário, para aplicação em 
pré-emergência de sulfometuron (18,7 $\mathrm{g} \mathrm{ha}^{-1}$ i.a.) + chlorimuron (18,7 $\mathrm{g} \mathrm{ha}^{-1}$ i.a.).

A aplicação de sulfometuron + chlorimuron, em pré ou pós-emergência, seguida da aplicação de glyphosate em pós-emergência, vem sendo utilizada no manejo de Amaranthus palmeri (Metzler, 2016) e Echinochloa crus-galli (Metzler e Ahumada, 2016), em soja $\mathrm{RR}^{\circledR} / \mathrm{STS}^{\circledR}$, em regiões produtoras de grãos na Argentina.

Os resultados obtidos no presente trabalho posicionam os herbicidas utilizados no manejo de picão-preto. Destaque para a associação tripla (sulfometuron + chlorimuron + glyphosate) que se apresentou eficaz no manejo das três plantas daninhas estudadas. Caracterizando-se assim como alternativa para o manejo de plantas daninhas em soja $\mathrm{RR}^{\circledR} / \mathrm{STS}^{\circledR}$.

\section{Conclusões}

A aplicação de sulfometuron (15 $\mathrm{g} \mathrm{ha}^{-1}$ i.a.) + chlorimuron (20 $\mathrm{g} \mathrm{ha}^{-1}$ i.a.) + glyphosate (960 $\mathrm{g} \mathrm{ha}^{-1}$ e.a.), foi eficaz no controle de amendoim-bravo e corda-de-viola. Os demais tratamentos não foram eficazes no controle destas plantas daninhas.

Todos os tratamentos herbicidas foram eficazes no controle em pós-emergência de picão-preto.

\section{Referências}

Albrecht, A.J.P.; Silva, A.F.M.; Albrecht, L.P.; Pereira, V.G.C.; Krenchinski, F.H.; Migliavacca, R.A.; Victoria Filho, R. Effect of sulfonylureas application on RR/STS soybean. Revista de Agricultura, v.92, n.1, p.37-49, 2017.

Atkinson, J.L.; Cross, R.B.; McCarty, L.B.; Estes, A.G. Control of American Burnweed (Erechtites hieraciifolia) in Bermudagrass Turf. Weed Technology, v.28, n.4, p.646-652, 2014. http:// dx.doi.org/10.1614/WT-D-14-00025.1.

Brasil. MAPA - Ministério da Agricultura Pecuária e Abastecimento. Sistemas de Agrotóxicos Fitossanitários. AGROFIT: consulta de ingrediente ativo. Brasília, 2017. Disponível em: $<$ http:// agrofit.agricultura.gov.br/agrofit_cons/principal_ agrofit_cons>. Acesso em: 04 set. 2017.

Constantin, J. Métodos de manejo. In: Oliveira Júnior, R.S.; Constantin, J.; Inoue, M.H. (Ed.). Biologia e manejo de plantas daninhas. Curitiba: Ominipax, 2011. p.67-78

Ferreira, D. Sisvar: a guide for its bootstrap procedures in multiple comparisons. Ciência e Agrotecnologia, v.38, n.2, p.109-112, 2014. http:// dx.doi.org/10.1590/S1413-70542014000200001.

Ferreira, E.A.; Matos, C.D.C.; Barbosa, E.A.; Melo, C.A.D.; Silva, D.V.; Santos, J.B. Aspectos fisiológicos de soja transgênica submetida à competição com plantas daninhas. Revista de Ciências Agrárias, v.58, n.2, p.115-121, 2015.

Forte, C.T.; Basso, F.J.M.; Galon, L.; Agazzi, L.R.; Nonemacher, F.; Concenço, G. Habilidade competitiva de cultivares de soja transgênica convivendo com plantas daninhas. Agrária, v.12, n.2, p.185-193, 2017. http://dx.doi.org/10.5039/ agraria.v12i2a5444.

Gazziero, D.L.P. Misturas de agrotóxicos em tanque nas propriedades agrícolas do Brasil. Planta Daninha, v.33, n.1, p.83-92, 2015. http:// dx.doi.org/10.1590/S0100-83582015000100010.

Green, J.M. Review of glyphosate and ALSinhibiting herbicide crop resistance and resistant weed management. Weed Technology, v.21, n.2, p.547-558, 2007. http://dx.doi.org/10.1614/ WT-06-004.1.

Heap, I. International Survey of Herbicide Resistant Weeds. 2018. Disponível em: $<$ http:// www.weedscience.org > . Acesso em: 28 fev. 2018.

Johanning, N.R.; Walters, S.A.; Young, B.G. Herbicides for control of volunteer horseradish (Armoracia rusticana) and potential carryover to subsequent horseradish production. Weed Technology, v.30, n.1, p.181-189, 2016. http:// dx.doi.org/10.1614/WT-D-15-00087.1. 
Kissmann, K.G.; Groth, D. Plantas infestantes e nocivas. São Paulo: BASF, 2007.

Li, Z.; Acker, R.V.; Robinson, D.E.; Soltani, N.; Sikkema, P.H. Halosulfuron tankmixes applied preplant incorporated for weed control in white bean (Phaseolus vulgaris L.). Canadian Journal of Plant Science, v.96, n.1, p.81-88, 2016. http:// dx.doi.org/10.1139/cjps-2015-0124.

Metzler, M. Amaranthus palmeri S. Watson: biología y manejo. Argentina: INTA, 2016. p.416. (Serie Extensión Digital INTA CRER, n.2).

Metzler, M.; Ahumada, M. Evaluación de herbicidas residuales preemergentes para el control de Echinochloa crus-galli en Entre Ríos. Argentina: INTA, 2016. p.1-14. (Serie de Extensión INTA Paraná, n.4).

Moreira, H.J.C.; Bragança, H.N.P. Manual de identificação de plantas infestantes. Campinas: FMC Agricultural Products, 2011. 1017p.

Nandula, V.; Poston, D.; Reddy, K.; Whiting, K. Response of soybean to halosulfuron herbicide. International Journal of Agronomy, v.2009, p.17, 2009. http://dx.doi.org/10.1155/2009/754510.

Nonemacher, F.; Galon, L.; Santin, C.O.; Forte, C.T.; Fiabane, R.C.; Winter, F.L.; et al. Associação de herbicidas aplicados para o controle de plantas daninhas em soja resistente ao glyphosate. Revista Brasileira de Herbicidas, v.16, n.2, p.142-151, 2017. http://dx.doi.org/10.7824/rbh.v16i2.529.

Olea, I. Malezas resistentes a glifosato en el Noroeste Argentino: situación actual y manejo. In: Rios, A. (Ed.). Viabilidad del glifosato en sistemas productivos sustentables. Montevideo: INIA, 2013. 176p.

Pagnoncelli, F.D.B.; Trezzi, M.M.; Brum, B.; Vidal, R.A.; Portes, Á.F.; Scalcon, E.L.; Machado, A. Morning glory species interference on the development and yield of soybeans. Bragantia, v.76, n.4, p.470-479, 2017. http://dx.doi. org/10.1590/1678-4499.2016.338.

Piasecki, C.; Rizzardi, M.A. Herbicidas aplicados em pré-emergência controlam plantas individuais e touceiras de milho voluntário $\mathrm{RR}^{\circledR} \mathrm{F} 2$ em soja? Revista Brasileira de Herbicidas, v.15, n.4, p.323-331, 2016. http://dx.doi.org/10.7824/rbh. v15i4.497.

Pimentel-Gomes, F.; Garcia, C.H. Estatística aplicada a experimentos agronômicos e florestais: exposição com exemplos e orientações para uso de aplicativos. Piracicaba: FEALQ, 2002. 309p.

Riar, D.S.; Norsworthy, J.K.; Steckel, L.E.; Stephenson, D.O.; Eubank, T.W.; Bond, J.; Scott, R.C. Adoption of best management practices for herbicide-resistant weeds in midsouthern united states cotton, rice, and soybean. Weed Technology, v.27, n.4, p.788-797, 2013. http:// dx.doi.org/10.1614/WT-D-13-00087.1.

Rodrigues, B.N.; Almeida, F.S. Guia de herbicidas. 6.ed. Londrina: Edição dos Autores, 2011. 697p.

Roso, A.C.; Vidal, R.A. Culturas resistentes aos herbicidas inibidores da enzima ALS: revisão de literatura. Pesticidas: Revista de Ecotoxicologia e Meio Ambiente, v.21, p.13-24, 2011.

Shaner, D.L. The impact of glyphosate-tolerant crops on the use of other herbicides and on resistance management. Pest Management Science, v.56, n.4, p.320-326, 2000. http://dx.doi.org/10.1002/ (SICI)1526-4998(200004)56:4<320::AIDPS125>3.0.CO;2-B.

Silva, A.F.M.; Albrecht, A.J.P.; Albrecht, L.P.; Victoria Filho, R.; Giovanelli, B.F. Application of post-emergence als inhibitor herbicides associated or not to glyphosate in RR/STS soybean. Planta Daninha, v.34, n.4, p.765-775, 2016. http://dx.doi. org/10.1590/s0100-83582016340400017.

Velini, D.E.; Osipe, R.; Gazziero, D.L.P. Procedimentos para instalação, avaliação e análise de experimentos com herbicidas. Londrina: SBCPD, 1995. 42p.

Zhou, Q.; Liu, W.; Zhang, Y.; Liu, K.K. Action mechanisms of acetolactate synthase-inhibiting herbicides. Pesticide Biochemistry and Physiology, v.89, n.2, p.89-96, 2007. http://dx.doi.org/10.1016/j. pestbp.2007.04.004. 